\title{
Finite-element simulation of aluminum temperature field and thermal profile in laser welding process
}

\author{
Ali Moarrefzadeh \\ Young Researchers Club, Mahshahr Branch, Islamic Azad University, Mahshahr, Iran \\ A_moarrefzadeh@yahoo.com, A.moarefzadeh@mahshahriau.ac.ir
}

\begin{abstract}
When a laser beam is irradiated on the surface of a material, the absorbed energy causes the heating, melting, and/ or evaporation of the material depending on the absorbed laser power density. The general condition of laser welding process is to create a pool of molten material (weld pool) at the overlapping work piece surfaces. There are two general approaches for laser welding processes. In this paper, the laser beam welding is studied and Aluminum temperature field is gained in this process. The thermal effect of laser beam that specially depends on the laser type and temperature field of it in work piece is the main key of analysis and optimization of this process, from which the main goal of this paper has been defined. Utilizing laser as a method to join plastic components is growing in popularity. There are two laser welding mechanisms, keyhole mode and conduction mode. Keyhole welding is widely used because it produces welds with high aspect ratios and narrow heat affected zones. However keyhole welding can be unstable, as the keyhole oscillates and closes intermittently. This intermittent closure causes porosity due to gas entrapment. Conduction welding, on the other hand, is more stable since vaporization is minimal and hence there is no further absorption below the surface of the material. Conduction welds are usually produced using low-power focused laser beams. This results in shallow welds with a low aspect ratio. In this work, high-power $\mathrm{CO}_{2}$ and $\mathrm{YAG}$ lasers have been used to produce laser conduction welds on $2 \mathrm{~mm}$ and $3 \mathrm{~mm}$ gauge AA5083 respectively by means of defocused beams. Full penetration butt-welds of and $3 \mathrm{~mm}$ gauges AA508 using this process have been produced. It has been observed that in this regime the penetration depth increases initially up to a maximum and then decreases with increasing spot size.
\end{abstract}

Keywords: Aluminum, Laser welding, Finite-Element, ANSYS.

\section{Introduction}

Laser welding and arc welding methods have been used in many industries for a long time, and their application is being continuously developed. Laser welding has many attractive features: laser beams can be focused to a very small diameter, and can melt only very small portions of materials. For this reason, laser welding can be conducted without affecting nearby materials, thus, the heat-affected zone (HAZ) is usually very small. This makes laser welding suitable for the precise welding of small parts. Laser beam power and profile can be adjusted or modified easily to make keyholes, thus, deep penetration welding is possible. There are many attributes of laser light that are uniquely suited for laser welding. A beam of laser light is monochromatic (single wavelength) and collimated (parallel) and hence can be focused down to a very small spot where the photon density is high enough to melt metals and alloys in a matter of milli-seconds. Laser wavelengths are typically identified by the laser source used to produce laser light. The most commonly used for pulsed welding is 1.064 micron Nd:YAG wavelength that has the option of being transmitted through an optical fiber. A new generation of lasers called fiber lasers also have similar wavelength where the laser light is produced in the fiber itself. Fiber lasers have the benefit of producing good beam quality and hence can have much longer working distance (distance between lens and work piece), of the order of ten inches as compared to about two inches for

Research article

(C)Indian Society for Education and Environment (iSee) conventional YAG lasers. Another source commercially available is the 10.64 micron $\mathrm{CO}_{2}$ laser, thought that is more often used for continuous wave welding rather than for pulsed welding.

The laser beam can travel through air or vacuum with minimal loss of energy. In some applications, the entire laser delivery hardware including focus head and fiber can be mounted inside a glove box where the environment is controlled for oxygen and water vapor contamination as is necessary for welding of Titanium. If required, the controlled environment can be produced in a more confined space with the laser energy being delivered through a quartz window.

Laser welding does not require that the part being welded be electrically conductive as is required for competing technologies including resistance welding, arc welding, and electron beam welding. Consequently, lasers have been used to weld all types of materials including metals, ceramics/glasses, and plastics. In welding of metals, all the laser energy is absorbed on the surface of the metal where as in glasses; part of the energy is absorbed on the surface while the remaining portion is absorbed in the bulk.

Welding of plastics is performed by facilitating absorption of laser energy at the weld interface either by using an absorptive layer or having one of the plastic be colored for preferential absorption.

Perhaps the most important aspect that has led to the growing use of lasers for welding is the ability to make 
spot welds. A laser beam focused down to a spot can heat, melt, and solidify metals in a matter of milli-seconds with minimal disturbance to adjoining volume of material and components. Consequently, laser spot welding is finding ever increasing applications in all segments of manufacturing including medical devices, sensors, batteries, and microwave enclosures. Along with growth in applications, there has been substantial improvement in laser power supply capabilities including closed-loop feedback and pulse shaping. As laser pulse welding is pushed to its limits in new and unique applications, it will be increasingly important to have good understating of the laser pulse and its effect on the parts being welded. This paper presents insights into the anatomy of a laser pulse and its effect on weld size, shape, residual stress, and defects. These fields' equations are solved together. Finally by writing codes with FSI (Fluid solid Interface) and SIMPLEC way, the process is optimized. For this simulation design parameter are introduced then

Fig. 1 Experiment set-up of the laser welding

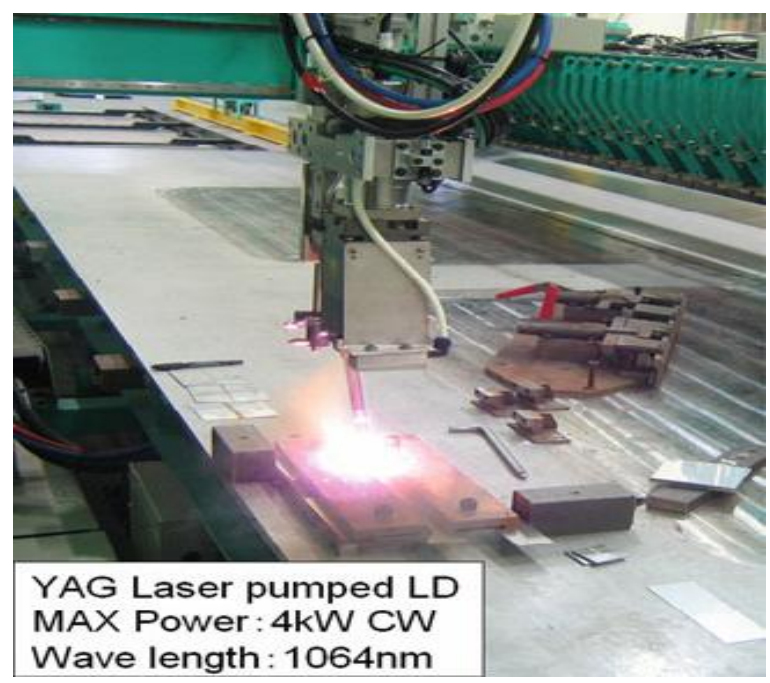

Fig. 2 Flow chart of the calculation

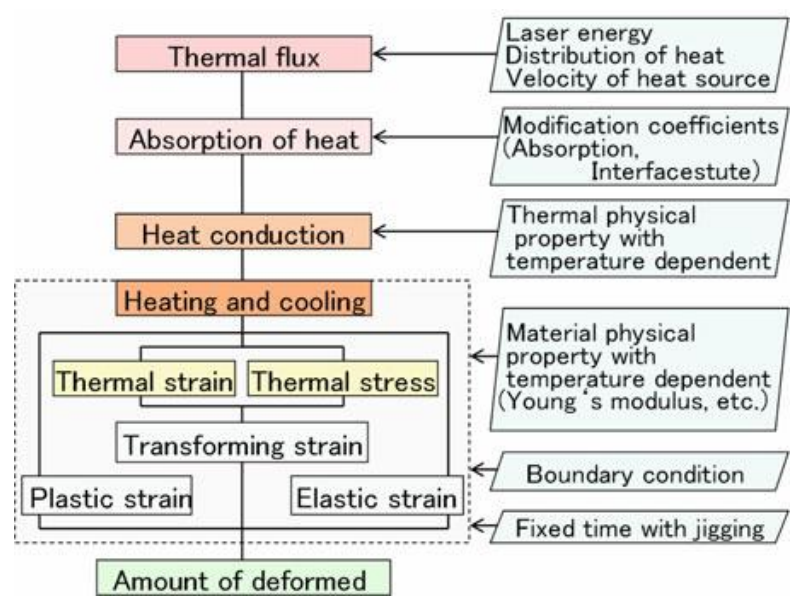

Research article

CIndian Society for Education and Environment (iSee) condition function parameters are introduced as function of design parameters. And objective function that is the temperature field of Aluminum, for achieving to the optimized fields is derived. That has suitable condition like cooling rate, the control of expanding HAZ (Heat Affected Zone) and optimization of consumed energy, for doing the process (Kyselica, 2005).

\section{Simulation of Bead on Plate}

This section describes the calculation model for laser welding. Fig. 1 shows the experimental set-up of the welding with an actual high power laser.

To clarify the distortion mechanisms of the thin plate and the thermal stress caused in the materials, the finite element method was used and 3-D no stationary elasto plasticity analysis was performed in which the laser heat source was emitted moving at a constant rate. In this calculation, radiation and convection were taken into account for contact heat transfer at the nodal points of the element. Fig.2 illustrates the flow chart of the simulation calculation. This simulation is basically a thermal analysis simulation using the finite element method but it obtains the temperature field by using a heat transfer equation which takes eradiation, convection and contact heat transfer into account. Then elastic-plastic stress distortion analysis was carried out by giving the thermal strain obtained from the temperature.

In this simulation, the laser heat source is replaced with a heat flux which was converted to heat quantity, and the temperature was calculated considering the ratio of beam absorption and the thermal property of the material.

Fig.3. Finite-Element Modeling
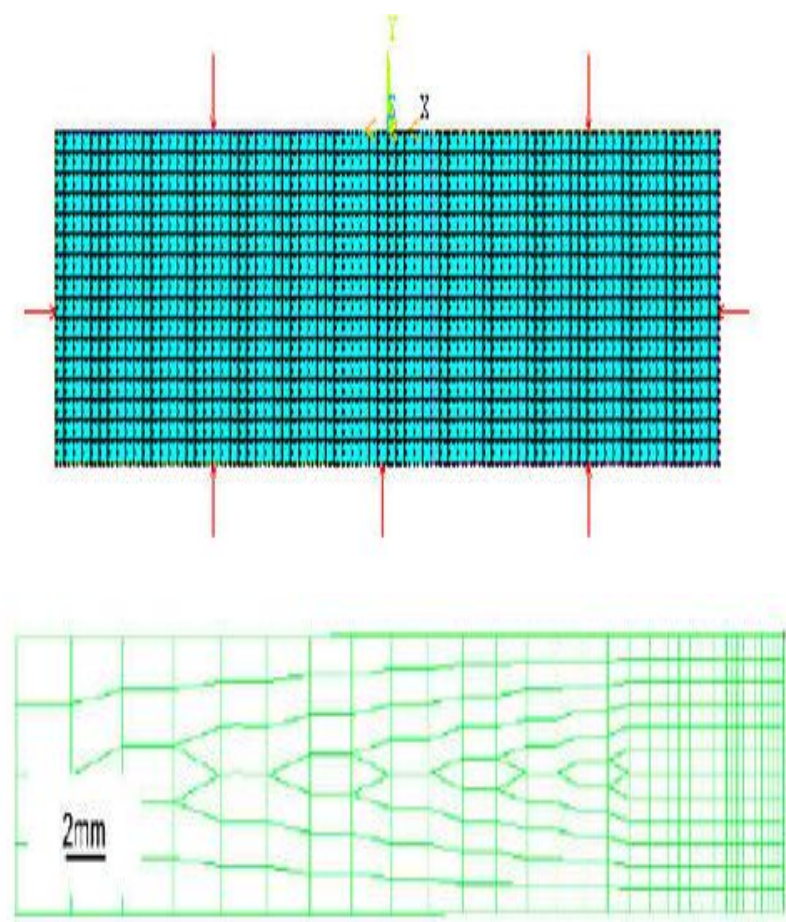

"Laser welding" http://www.indjst.org
Ali Moarrefzadeh Indian J.Sci.Technol. 
Fig.4. Inert gas shielding configurations used for top of welds.
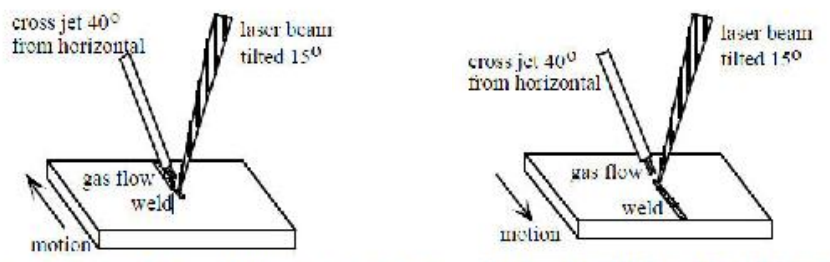

(a) cross jet pointed ac leading edge of weld pool

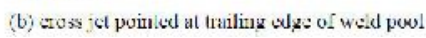

Fig.5. Joint shape and jig system used in experiments. a. Tube formed prior to welding process. b. Jig used in experiments.

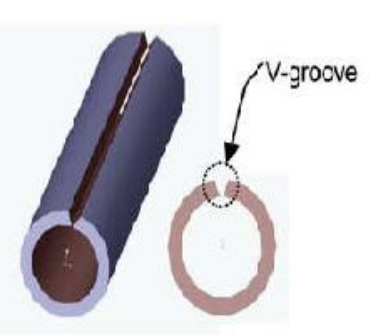

a Tube formed prior to welding process

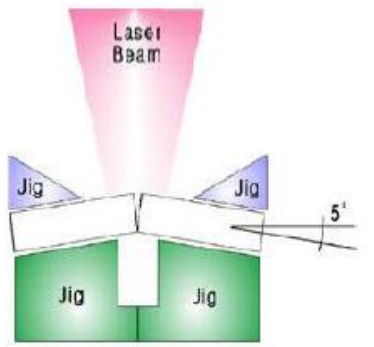

b Jig used in experiments

Fig.6. Schematic diagram of the welding technique
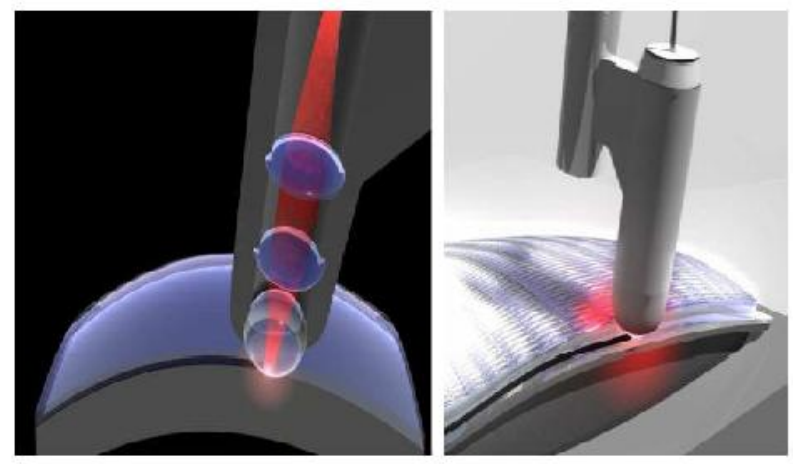

Moreover, stress, thermal strain, transforming strains, plastic strain and elastic strain were determined by using the elastic constants of the materials such as the Young's modulus and the Poisson's ratio. Finally the distortion amount of the entire plate was calculated. Also, temperature dependence was taken into account for the physical properties of the materials and the latent heat was considered as well in the molten state.

\section{Numerical simulation}

Finite elements simulations are done in 3 steps with the main pieces: 1) Modeling by FEMB, 2) The thermal study and processing and 3) Post-Processing result of analysis by Ansys software.

Numerical solution

-In numerical method that is done with ANSYS code the finite element (SIMPLEC) is used.

- In numerical solution by considering the interface solid and fluid, the FSI is used.

Finite element modeling

Research article

CIndian Society for Education and Environment (iSee)
"Laser welding"

http://www.indjst.org
Vol. 5 No. 8 (August 2012) ISSN: 0974- 6846

Fig. 7. Measurement result of distortion in the case of bead-on-plate

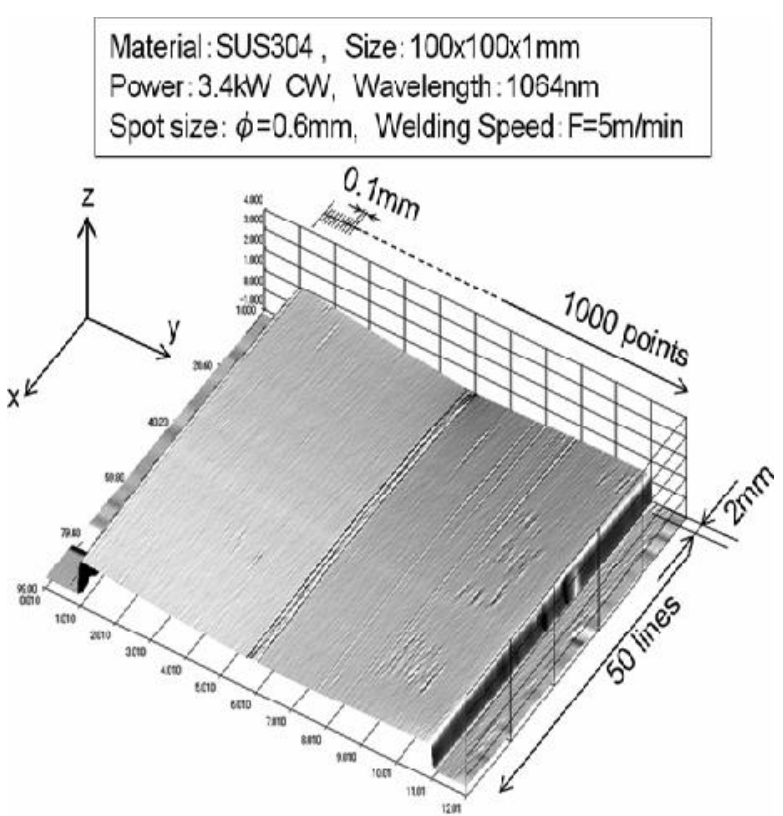

Keyence Corporation. : LK-G (CCD Laser Displacement Sensor )

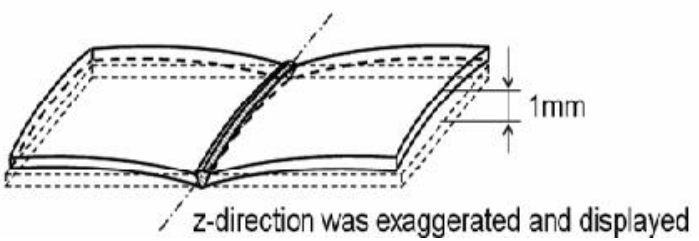

Fig. 8. Result of deformation in bead-on-plate

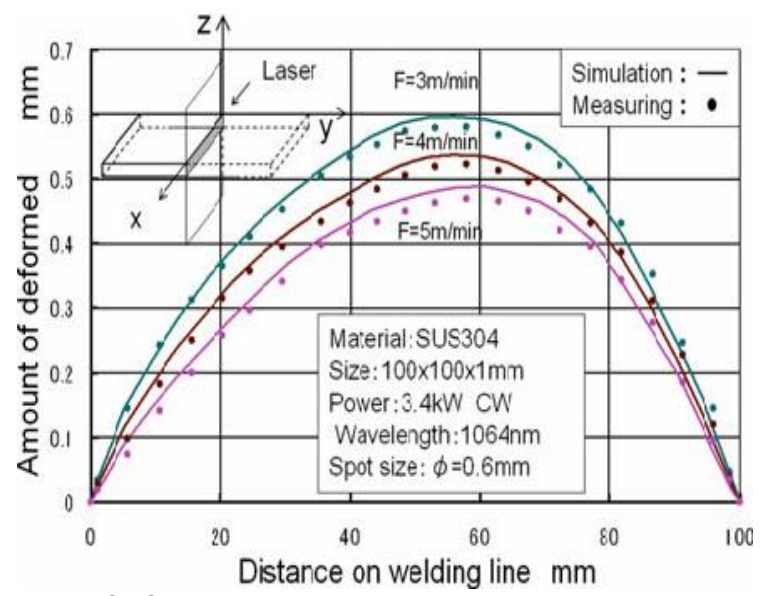

In Fig.3, finite element model is shown. The model is 2-dimensional and axis symmetric. In this model, the method of meshing is manual and the thickness of each mesh is $0.00005 \mathrm{~m}$. This meshing is done in a way that solid and fluid mesh is exactly fixed. For meshing of solid field (Aluminum) by considering the study of thermal field, from the thermal elements set, we chose the PLANE55 type. As axis symmetric element with conduction property, this element has 4 nodes with one degree of freedom. This element has mesh moving property as well for laser field use FLUID142 element and is suitable for Ali Moarrefzadeh Indian J.Sci.Technol. 
Fig.9. Moving picture simulation of the distortion in the case of bead-on-plate

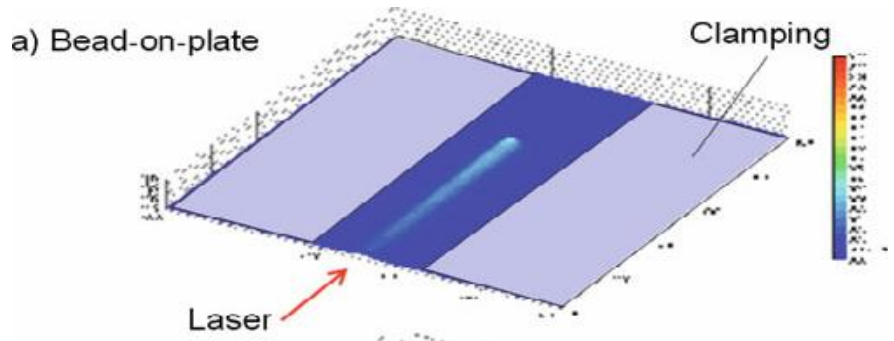

b) Clamp removed

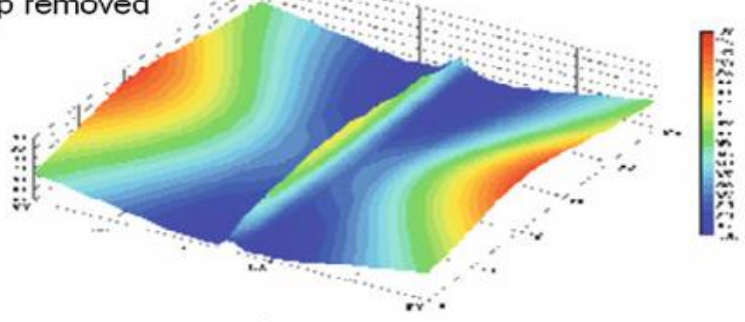

c) After cooling

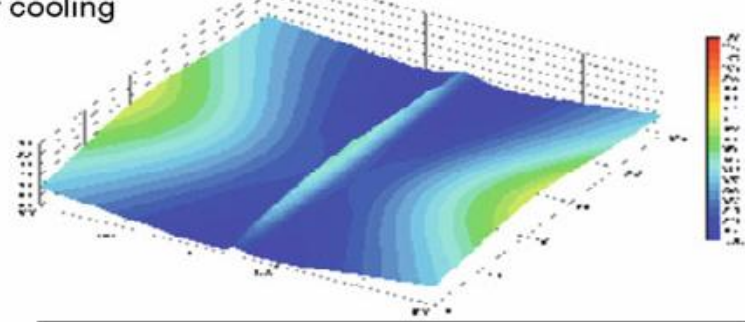

Material: SUS304, Size: $100 \times 100 \times 1 \mathrm{~mm}$

Power: $3.4 \mathrm{~kW}$, Welding Speed: $F=5 \mathrm{~m} / \mathrm{min}$

transient thermal modeling. Besides, this element has thermal energy transmitting property.

The differential Eqs. (1)-(4) are solved iteratively by the SIMPLEC numerical procedure:

For boundary condition of fluid field:

$$
\begin{aligned}
& \int_{\Omega} \partial P\left[\frac{1}{C^{2}} \ddot{P}+(\nabla)^{Y} \nabla P\right] d \Omega+ \\
& \int_{T_{1}} \partial P n^{T} \ddot{u} d T+\int_{T_{3}} \partial P \frac{1}{g} \ddot{P} d T=0
\end{aligned}
$$

For boundary condition of solid field:

$$
\begin{aligned}
& \int_{\Omega} \partial u\left[P_{s} \ddot{u}+S^{T} D S u\right] d \Omega- \\
& \int_{T_{1}} \partial u^{T} \bar{t} d T=0
\end{aligned}
$$

Heat transfer equation:

For conduction :

$$
q_{X}=-k_{x x} \frac{d T}{d x}
$$

$\frac{\partial}{\partial x}\left(k_{x x} \partial T / \partial x\right)+\partial / \partial y\left(k_{y y} \partial T / \partial y\right)+Q=0$

\section{Gas Shielding Effects}

The different gas shielding configurations illustrated in Fig. 4 were used to determine top shielding effects on weld appearance. The slightly different angles were necessitated by constraints in the experimental system and fixturing limitations but do not produce significant differences in results. Beam focus was located at the work piece surface. The different cross jet configurations affect the plasma formation over the weld. The leading edge configuration tends to blow plasma to the to-bewelded location while the trailing edge and transverse direction configurations blow the plasma away from the to-be-welded location. The presence of plasma tends to absorb and defocus the beam. Unpublished tests by the authors on Aluminium parts have shown that weld penetration is decreased using the leading edge configuration. The results obtained for Aluminium using the TEM20 beam at $5.1 \mathrm{~kW}$ and a weld speed of $12.7 \mathrm{~m} / \mathrm{s}$ also show a decreased penetration for the leading edge case.

\section{Semi-quantitative analysis}

Location of Beam Focus

Most of the data reported here are obtained using a beam focus position at or above $(\sim 2.5 \mathrm{~mm})$ the workpiece surface.. found that using beam defocusing produced better welds and avoided hole formation but beam irradiances were not measured precisely. Park et al. located beam focus below the surface and obtained good welds. We have observed that locating the beam focus inside the weld increased the welding efficiency in that substantially higher weld speeds (16.9 instead of 12.7 $\mathrm{m} / \mathrm{s}$ ) can be used to obtain the same penetration if the irradiance is controlled. The effect of high irradiance on weld quality will be discussed elsewhere (Matsuda et al.,1988).

Weld Quality

Laser welding of Aluminium tends to produce a higher degree of spatter than for the case of Aluminium. A higher cross-flow was necessary to prevent spatter on the optics. High irradiances also tend to produce more spatter and lower weld surface quality for Aluminium.

The spot sizes at focus were 200 and $400 \mu \mathrm{m}$ for the TEM00 and TEM20 beams respectively. Beam focus was located at the workpiece surface. The voids/porosities in the weld cross sections tend to be relatively small with a few larger voids. All voids were smaller than $0.1 \mathrm{~mm}$. The welds obtained with the TEM00 beam tend to have higher porosity. The irradiances used here were very similar for both beam modes. The narrower welds higher porosity could result from the smaller surface area and more constrained convection of the weld pool for expulsion of gases produced. All weld cross sections examined showed some degree of porosity regardless of edge 
preparation. Dry milled or wire-brushed edges had similar levels of porosity.

The tube seam line is slightly opened, and this gap plays a roll as a V-groove in butt welding joints, as in Fig.

Fig. 10. Solid (5182 Aluminum alloy) temperature field

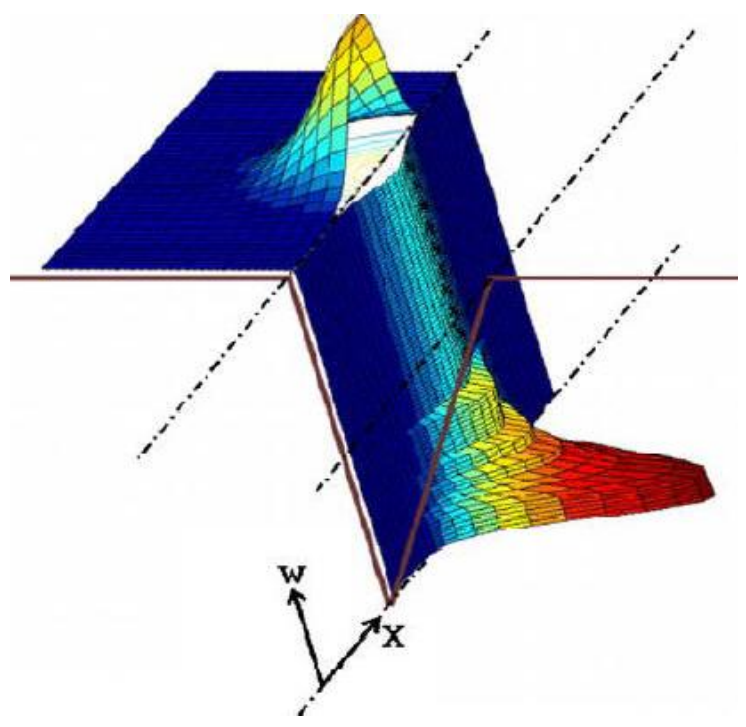

Fig. 11. Distribution of absorbed intensity.

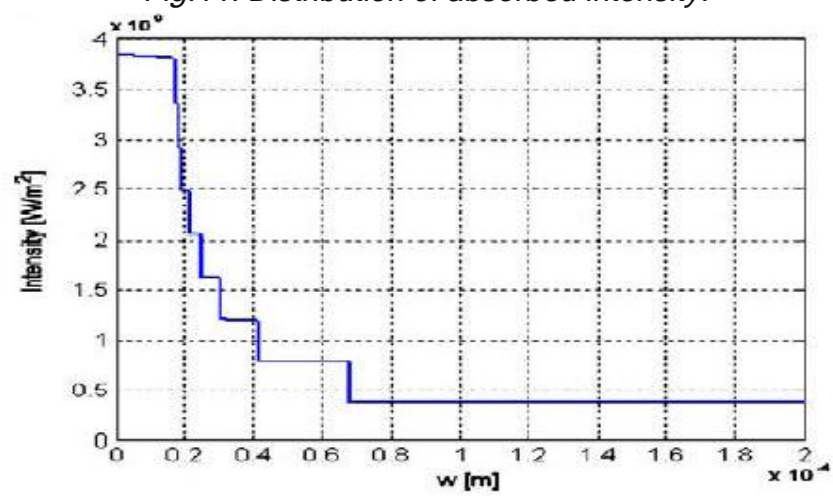

5a. This V-groove enhances absorption of the laser beam by the mechanism of multiple reflections within it. To simulate this process in laboratory experiments, the jig

Fig. 12. Conduction weld for $3 \mathrm{~mm}$ gauge AA5083 using YAG laser; Average power $=2000 \mathrm{~W} ;$ Weld speed $=600 \mathrm{~mm} / \mathrm{min}$

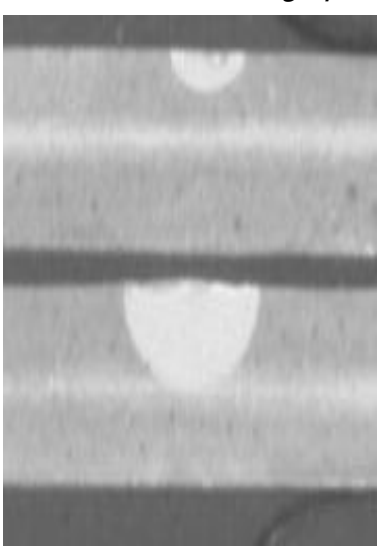

Research article

COIndian Society for Education and Environment (iSee)

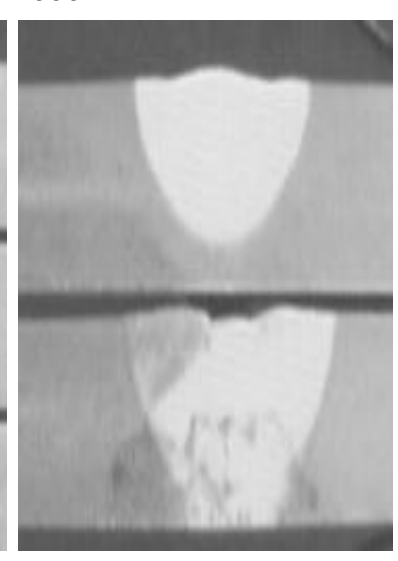

"Laser welding" http://www.indjst.org makes two strips be positioned slightly inclined and brought into contact with each other, like conventional butt joints. The cross section of the designed jig is shown in Fig. 5b, which shows two strips inclined at an angle of $5^{\circ}$, and the laser beam is irradiated onto the $\mathrm{V}$-groove joint. Therefore, the seam welding of small-diameter pipe can be successfully simulated by butt joint welding of thin stainless steel strips.

Welding concept for three-dimensional joining applications

A new concept was recently introduced to eliminate the technical limitations encountered in the use of clamping systems and to facilitate the use of laser for three dimensional joining. With this new process the contact pressure required for the joining process is constantly regulated to act dynamically, selectively, perpendicularly and precisely at the desired joining area. The welding concept essentially works on the contour welding principle, whereby the laser spot follows a contour and the component is sequentially welded.

A laser spot is focused on the joining plane by means of an air bearing, frictionless, rotating glass sphere as shown in Fig. 6 . The glass sphere lens serves as a mechanical pressing tool applied perpendicularly at each point on the joining plane. This ensures that the laser beam is only incident at the site where the contact pressure is applied. This process concept offers the possibility of applying the necessary contact pressure concurrent with the laser beam being continuously moved along a welding contour. The air bearing glass sphere lens is fitted in a robust and compact processing head together with the optical fiber connector and other optical systems and process monitoring sensors (Matsuda et al.,1988).

Fig.7 shows an example of the result of a measurement that uses this measuring instrument. The simulation result of the distortion was examined, in which the plate was assumed to be cooled down sufficiently after the heat source passed over.

Fig. 8 shows the distortion close to the welding line calculated for bead-on plate. In this calculation, a plate with $1 \mathrm{~mm}$ thickness was processed with the same power level as the above, setting the welding speed in the range of 3-5 $\mathrm{m} / \mathrm{min}$. In laser welding, the slower the welding speed gets, the larger the swelling becomes near the center upward.

Furthermore, the amount of distortion differs between penetration welding and non-penetration welding. The result shows that the slower the welding speed gets, the larger the distortion amount becomes.

In addition, the simulation and the actual experiment compare reasonably well. The solid line corresponds to the simulation, and the points refer to the measurement result in the experiment. Indian J.Sci.Technol. 


\section{Result and discussions}

Fig.9 shows two-dimensional distributions of the temperature and flow velocity in 3 case thickness. Details provided in this work on Aluminium alloy together with results obtained by other researchers on Aluminium alloys reveal that laser beam welding of Aluminium alloys has limitations in obtaining the desired weld strength and formability required for automotive applications. In particular, for $1.8 \mathrm{~mm} 5182$ Aluminium alloy samples with shear cut edges, butt welds obtained with beams achieved ultimate tensile strength of $251 \mathrm{MPa}$, elongation of $10.4 \%$ and $75 \%$ of desired formability in terms of dome height. results had corresponding values of $190 \mathrm{MPa}$, $5.4 \%$ and $77 \%$ respectively. Conclusions for solid (5182 Aluminium alloy) temperature field, completely and showing the heat transfer way between laser beam and shielding gas and between shielding gas and environment with temperature and velocities for different gases is completely shown in Fig. 10 .

Also, Fig.11 shows the final 3D distribution of absorbed intensity. Conduction welds were produced using high-power defocused laser beams on $2 \mathrm{~mm}$ and $3 \mathrm{~mm}$ gauge AA5083. Fig.12 shows micrographs of conduction welds made from $3 \mathrm{~mm}$ gauge AA5083. The numbering is done in order of increasing spot radius. The expected hemispherical shape characteristic of conduction welds was obtained. The variation of the penetration depth with increasing spot radius or distance from the focus becomes apparent on close examination. In all cases it was found that the aspect ratios (penetration depth divided by weld width) of the welds were less than one. Penetration depth/spot radius curves plotted showed an increase (up to a maximum) and then a decrease of penetration depth with increase in spot radius in the conduction weld regime. It can be attributed to the interplay between decreasing power density and increasing interaction time, as the spot radius becomes larger.

\section{Conclusion}

According to result achieved from coupling field analysis and comparison of temperature field and heat transfer in laser beam welding with other process, it can be derived that the laser welding has more arc length, concentration of higher energy, better stability and more welding depth to width (specially for Aluminum tube). Laser welding is emerging as an important welding technique in plastics processing. The diverse fields of application always call for new techniques and innovative problem solving approaches. Though various laser transmission welding techniques have been introduced that complement conventional joining methods, the innovative potential of laser transmission welding has yet to be fully exploited.

By virtue of its precise and controllable application of the necessary joining force this process produces an optically perfect welding seam, which is of crucial importance in the manufacture of decorative components.
A typical example is the manufacture of automobile headlights or tail lights, which require a three-dimensional welding seam. Semi-quantitative and experimental investigations into laser conduction welding (LCW) of AA5083 using high-power defocused CO2 and YAG laser beams reveal that penetration depth increases up to a maximum and then decreases with increasing spot radius. More specifically, Semi-quantitative analysis of the LCW process shows that the penetration depth increases with increase in spot radius when the surface temperature remains at boiling temperature. This trend is reversed when the boiling temperature is no longer sustained due to a further increase in the spot radius. This confirms that the penetration depth/spot radius variation occurs well within the conduction-welding regime, as ideally there should be no vaporization during LCW. - It is suggested that this phenomenon can be attributed to (i) the interplay between decreasing power density and increasing interaction time, (ii) the laser beam distribution and (iii) pre-heating due to the interaction of the outer fringes of the laser beam with the work piece.

This process may offer an alternative method of welding resulting in slower rates of cooling which may enhance weld bead properties of traditionally difficult materials such as aluminum alloys used in the aerospace industry. Full penetration laser conduction welds produced on such materials by means of high-power defocused laser beams could reduce problems encountered with keyhole welds thereby greatly reducing if not eliminating keyhole defects.

\section{Acknowledgments}

This paper was extracted from a research project entitled Finite-Element simulation of Aluminum temperature field in Laser Welding. Financial assistance from Young Researchers Club, Islamic Azad University, Mahshahr Branch is gratefully acknowledged

\section{References}

1. Ali Moarrefzadeh (2012) Study of heat affected zone for choosing suitable shielding and nozzle gas in plasma arc welding Indian J.Sci.Technol. 5(4), 26192626.

2. Matsuda J, Utsumi A, Katsumura M, Hamasaki M and Nagata S (1988) TIG or MIG arc augmented laser welding of thick Mild steel plate. Join Matter. 1(1),3134.

3. Moarrefzadeh A (2010) Numerical analysis of $\alpha$ phase stainless steel thermal profile in keyhole plasma arc welding (PAW) Process IREME J. pp.5764

4. Moarrefzadeh A (2010) Numerical simulation of temperature fiel by Plasma arc welding Process in stainless steel IREMO J. 3, 101-107.

5. Moarrefzadeh, A (2010) Choosing suitable shielding gas for thermal optimization of GTAW process. IREME J. pp: $748-754$.

6. Ushio $\mathrm{M}$ and Terasaki $\mathrm{H}$ (2005) Plasma arc keyhole welding. Welding J. pp. 331-340.
Research article

CIndian Society for Education and Environment (iSee)
"Laser welding" http://www.indjst.org
Ali Moarrefzadeh Indian J.Sci.Technol. 